\title{
Modal description of longitudinal space-charge fields in pulse-driven free-electron devices
}

\author{
Yu. Lurie* and Y. Pinhasi \\ Department of Electrical and Electronic Engineering, Ariel University Center of Samaria, Ariel, Israel
} (Received 31 January 2010; published 12 May 2010)

\begin{abstract}
In pulsed-beam free-electron devices, longitudinal space-charge fields result in collective effects leading to an expansion of short electron bunches along their trajectory. This effect restricts an application of intense ultrashort electron pulses in free-electron radiation sources. A careful theoretical treatment is required in order to achieve an accurate description of the self-fields and the resulted electron beam dynamics. In this paper, longitudinal space-charge fields are considered in the framework of a threedimensional, space-frequency approach. The model is based on the expansion of the total electromagnetic field (including self-fields) in terms of transverse eigenmodes of the (cold) cavity, in which the field is excited and propagates. The electromagnetic field, originally obtained in the model as a solution of the wave equation, is shown to satisfy also Gauss's law. We applied the theory to derive an analytical expression for the longitudinal electric field of a pointlike charge, moving along a waveguide at a constant velocity. This enables consideration and study of the role played by different terms of the resulted expressions, such as components arising from forward and backward waves, propagating waves, and under cutoff frequencies, and so on. Possible simplifications in evaluation of longitudinal space-charge fields are discussed.
\end{abstract}

DOI: 10.1103/PhysRevSTAB.13.050701

PACS numbers: 41.60.Cr, 52.59.Rz, 41.85.Ja

\section{INTRODUCTION}

Development of laser induced photocathodes and of electron bunch compression techniques enables application of intensive, ultrashort electron pulses in free-electron radiation sources. In free-electron lasers, a highly efficient superradiant emission can be achieved with such pulses, providing a strong coherent radiation with intensity being proportional to the pulse charge squared [1]. However, space-charge fields give rise to microbunching instabilities, restricting applications of intense ultrashort electron pulses [2-4]. In most electron devices, the electron beam is kept focused along the beam line so as the transverse components of the space-charge forces are compensated. The residual longitudinal space-charge field causes an expansion of short electron pulses along their trajectory. In a finite cross-section beam, there is a fringing field near the edges of the beam, which causes a decrease in the axial self-field. When the e-beam propagates in a waveguide, this reduction effect is even stronger due to the presence of conducting walls as shown schematically in Fig. 1.

In the present work, the self-fields are considered in the framework of a three-dimensional, space-frequency approach $[5,6]$. The model is based on the expansion of the total electromagnetic field in terms of transverse eigenmodes of the (cold) cavity, in which the field is excited and propagates. This approach has been applied for the analysis of wideband interactions in free-electron lasers operating in the linear and nonlinear regimes $[7,8]$. Based on this modal expansion, the three-dimensional theory for electro-

*ylurie@ariel.ac.il magnetic field and plasma wave propagation in a waveguide filled with a continuous electron beam was developed [9-11]. It is now extended to describe spacecharge fields arising in a pulsed-beam configurations.

The electromagnetic field, originally obtained in the model as a solution of the wave equation for an uniform waveguide, is shown to satisfy also Gauss's laws for electric and magnetic fields. The longitudinal electric field was found in the model analytically for a pointlike charge, moving along a waveguide at a constant velocity. This enables analysis of the role played by the different terms of the resulted expression for the longitudinal electric field, i.e., the components arising from forward and backward waves, above and under cutoff frequencies, and so on. Possible approximations for a simplified evaluation of longitudinal space-charge fields in a pulsed-beam device are discussed.

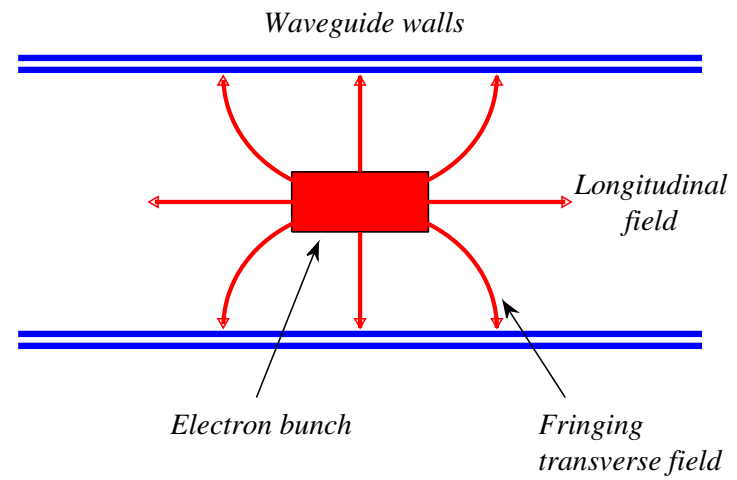

FIG. 1. (Color) Schematic illustration for fringing field. 


\section{MODAL PRESENTATION OF ELECTROMAGNETIC FIELD IN THE FREQUENCY DOMAIN}

In the approach, transverse components of the total electromagnetic field are expanded in the positivefrequency domain in terms of a complete set of transverse forward and backward eigenmodes of the medium in which the field is excited and propagates:

$$
\begin{aligned}
\tilde{\mathbf{E}}_{\perp}(\mathbf{r}, f) & =\sum_{ \pm q} C_{ \pm q}(z, f) e^{\mp j k_{z q} z} \tilde{\mathbf{E}}_{ \pm q \perp}\left(\mathbf{r}_{\perp}\right) \\
\tilde{\mathbf{H}}_{\perp}(\mathbf{r}, f) & =\sum_{ \pm q} C_{ \pm q}(z, f) e^{\mp j k_{z q} z} \tilde{\mathbf{H}}_{ \pm q \perp}\left(\mathbf{r}_{\perp}\right),
\end{aligned}
$$

where $C_{q}(z, f)$ is the scalar amplitude of the $q$ th mode with electric field $\tilde{\mathbf{E}}_{q \perp}\left(\mathbf{r}_{\perp}\right)$ and magnetic field $\tilde{\mathbf{H}}_{q \perp}\left(\mathbf{r}_{\perp}\right)$ profiles, and the axial wave number is

$$
k_{z q}(f)= \begin{cases}\sqrt{k^{2}-k_{\perp q}^{2}}, & k>k_{\perp q} \text { (propagating) } \\ -j \sqrt{k_{\perp q}^{2}-k^{2}}, & k<k_{\perp q} \text { (cutoff). }\end{cases}
$$

Here $k=w / c$ and $c=1 / \sqrt{\epsilon \mu}$ is the velocity of light.

Expressions for the longitudinal component of the electric and magnetic fields are obtained after substitution of the modal representation (1) into Maxwell's curl equations, where the electric current density $\tilde{\mathbf{J}}(\mathbf{r}, f)$ of the source electron beam is introduced:

$$
\begin{aligned}
\tilde{E}_{z}(\mathbf{r}, f) & =\sum_{ \pm q} C_{ \pm q}(z, f) e^{\mp j k_{z q} z} \tilde{\mathcal{E}}_{ \pm q z}\left(\mathbf{r}_{\perp}\right)-\frac{1}{j w \epsilon} \tilde{J}_{z}(\mathbf{r}, f) \\
\tilde{H}_{z}(\mathbf{r}, f) & =\sum_{ \pm q} C_{ \pm q}(z, f) e^{\mp j k_{z q} z} \tilde{\mathcal{H}}_{ \pm q z}\left(\mathbf{r}_{\perp}\right) .
\end{aligned}
$$

In the time domain, the electromagnetic field is given as inverse Fourier transform of (1) and (3):

$$
\begin{aligned}
& \mathbf{E}(\mathbf{r}, t)=\Re\left\{\int_{0}^{\infty} \tilde{\mathbf{E}}(\mathbf{r}, f) e^{+j w t} d f\right\} \\
& \mathbf{H}(\mathbf{r}, t)=\Re\left\{\int_{0}^{\infty} \tilde{\mathbf{H}}(\mathbf{r}, f) e^{+j w t} d f\right\} .
\end{aligned}
$$

Evolution of the amplitudes of the excited modes is described by a set of coupled differential equations of the first order:

$$
\begin{aligned}
\frac{d}{d z} C_{ \pm q}(z, f)= & \mp \frac{1}{2 \mathcal{N}_{q}} e^{ \pm j k_{z q} z} \\
& \times \iint\left[\left(\frac{Z_{q}}{Z_{q}^{*}}\right) \tilde{\mathbf{J}}_{\perp}(\mathbf{r}, f) \pm \hat{\mathbf{z}} \tilde{J}_{z}(\mathbf{r}, f)\right] \\
& \cdot \tilde{\mathcal{E}}_{q}^{*}\left(\mathbf{r}_{\perp}\right) d \mathbf{r}_{\perp} .
\end{aligned}
$$

The field amplitude of each mode is normalized via the complex Poynting vector:

$$
\mathcal{N}_{q}=\iint\left[\tilde{\mathbf{E}}_{q \perp}\left(\mathbf{r}_{\perp}\right) \times \tilde{\mathbf{H}}_{q \perp}^{*}\left(\mathbf{r}_{\perp}\right)\right] \cdot \hat{\mathbf{z}} d \mathbf{r}_{\perp}
$$

and the mode impedance is

$$
Z_{q}= \begin{cases}\sqrt{\frac{\mu}{\epsilon}} \frac{k}{k_{z q}}=w \mu / k_{z q} & \text { for TE modes } \\ \sqrt{\frac{\mu}{\epsilon}} \frac{k_{z q}}{k}=k_{z q} / w \epsilon & \text { for TM modes. }\end{cases}
$$

The spectral energy distribution of the electromagnetic field can also be expressed in terms of the excitation coefficients $C_{q}(z, f)$ of propagating and cutoff modes:

$$
\begin{aligned}
\frac{d \mathcal{W}(z)}{d f}= & \sum_{\substack{q \\
\text { propagating }}} \frac{1}{2}\left[\left|C_{+q}(z, f)\right|^{2}-\left|C_{-q}(z, f)\right|^{2}\right] \Re\left\{\mathcal{N}_{q}\right\} \\
& +\sum_{\substack{q \\
\text { culoff }}} \mathfrak{s}\left\{C_{+q}(z, f) C_{-q}^{*}(z, f)\right\} \mathfrak{S}\left\{\mathcal{N}_{q}\right\} .
\end{aligned}
$$

Equations (1) and (3)-(5) define the electromagnetic field in a waveguide as a solution of the wave equation for an uniform waveguide, obtained from Maxwell's curl equations. The divergence of the magnetic field can be presented in the frequency domain in the form

$$
\begin{aligned}
\nabla \cdot \tilde{\mathbf{H}}(\mathbf{r}, f) & =\nabla_{\perp} \cdot \tilde{\mathbf{H}}_{\perp}(\mathbf{r}, f)+\frac{\partial}{\partial z} \tilde{\mathbf{H}}_{z}(\mathbf{r}, f) \\
& =-\sum_{q} \frac{\left\langle\tilde{\mathcal{E}}_{q_{z}} \mid \tilde{\mathbf{J}}_{z}\right\rangle}{\mathcal{N}_{q}} \tilde{\mathcal{H}}_{q_{z}}\left(\mathbf{r}_{\perp}\right)
\end{aligned}
$$

where we define

$$
\left\langle\tilde{\mathcal{E}}_{q_{z}} \mid \tilde{\mathbf{J}}_{z}\right\rangle \equiv \iint \tilde{\mathbf{J}}_{z}(\mathbf{r}, f) \cdot \tilde{\mathcal{E}}_{q_{z}}^{*}\left(\mathbf{r}_{\perp}\right) d \mathbf{r}_{\perp} .
$$

Since one of the longitudinal field components, $\tilde{\mathcal{E}}_{q_{z}}\left(\mathbf{r}_{\perp}\right)$ or $\tilde{\mathcal{H}}_{q_{z}}\left(\mathbf{r}_{\perp}\right)$, is always equal to zero, i.e. $\tilde{\mathcal{E}}_{q_{z}}\left(\mathbf{r}_{\perp}\right)=0$ for TE modes and $\tilde{\mathcal{H}}_{q_{z}}\left(\mathbf{r}_{\perp}\right)=0$ for TM modes, the Gauss's law for the magnetic field

$$
\nabla \cdot \mathbf{H}(\mathbf{r}, t)=0
$$

is also satisfied.

The divergence of the electric field can be expressed as follows:

$$
\begin{aligned}
\nabla \cdot \tilde{\mathbf{E}}(\mathbf{r}, f) & =\nabla_{\perp} \cdot \tilde{\mathbf{E}}_{\perp}(\mathbf{r}, f)+\frac{\partial}{\partial z} \tilde{\mathbf{E}}_{z}(\mathbf{r}, f) \\
& =-\sum_{q} i_{q}(z) \tilde{\mathcal{E}}_{q_{z}}\left(\mathbf{r}_{\perp}\right)-\frac{1}{j w \boldsymbol{\epsilon}} \frac{\partial}{\partial z} \tilde{J}_{z}(\mathbf{r}, f),
\end{aligned}
$$

where

$$
i_{q}(z) \equiv \frac{1}{\mathcal{N}_{q}}\left(\frac{Z_{q}}{Z_{q}^{*}}\right) \iint \tilde{\mathbf{J}}_{\perp}(\mathbf{r}, f) \cdot \tilde{\mathcal{E}}_{q_{\perp}}^{*}\left(\mathbf{r}_{\perp}\right) d \mathbf{r}_{\perp} .
$$

Note that the summation in (12) actually includes only forward TM modes. Substituting $\tilde{\mathcal{E}}_{q_{z}}\left(\mathbf{r}_{\perp}\right)$ according to the relation

$$
\tilde{\mathcal{E}}_{q_{z}}\left(\mathbf{r}_{\perp}\right)=\frac{1}{j k_{z q}} \nabla_{\perp} \cdot \tilde{\mathcal{E}}_{q_{\perp}}\left(\mathbf{r}_{\perp}\right),
$$


the first term in (12) can be rewritten as follows:

$$
\begin{aligned}
-\sum_{q} i_{q}(z) \tilde{\mathcal{E}}_{q_{z}}\left(\mathbf{r}_{\perp}\right) & =-\frac{1}{j k_{z q}} \nabla_{\perp} \cdot \sum_{q} i_{q}(z) \tilde{\mathcal{E}}_{q_{\perp}}\left(\mathbf{r}_{\perp}\right) \\
& =-\frac{1}{j w \epsilon} \nabla_{\perp} \cdot \tilde{\mathbf{J}}_{\perp}(\mathbf{r}, f) .
\end{aligned}
$$

Using this result, the electric field is shown to satisfy the Gauss's law:

$$
\nabla \cdot \tilde{\mathbf{E}}(\mathbf{r}, f)=-\frac{1}{j w \epsilon} \nabla \cdot \tilde{\mathbf{J}}(\mathbf{r}, f)=\frac{\tilde{\rho}(\mathbf{r}, f)}{\epsilon},
$$

while applying the continuity equation

$$
\nabla \cdot \tilde{\mathbf{J}}(\mathbf{r}, f)=-j w \tilde{\rho}(\mathbf{r}, f),
$$

where $\tilde{\rho}(\mathbf{r}, f)$ is the charge density in the positivefrequency domain.

\section{ONE-DIMENSIONAL LONGITUDINAL TERM}

The last term in Eq. (3) introduces the one-dimensional, longitudinal field. In the time domain, the term corresponds to the field given by

$$
\begin{aligned}
\Delta E_{z}(\mathbf{r}, t) & =-\Re\left\{\int_{0}^{\infty} \frac{1}{j w \epsilon} \tilde{J}_{z}(\mathbf{r}, f) e^{+j w t} d f\right\} \\
& =\frac{\sigma_{>}(\mathbf{r})-\sigma_{<}(\mathbf{r})}{2 \epsilon},
\end{aligned}
$$

where

$$
\sigma_{>}(\mathbf{r}, t) \equiv \int_{t}^{+\infty} J_{z}(\mathbf{r}, \tau) d \tau
$$

and

$$
\sigma_{<}(\mathbf{r}, t) \equiv \int_{-\infty}^{t} J_{z}(\mathbf{r}, \tau) d \tau
$$

are the corresponding charge densities per unit square, originating from all the charges crossing the point $\mathbf{r}=$ $\left(\mathbf{r}_{\perp}, z\right)$ before and after the moment $t$. Within the transverse cross section of the electron beam pulse, these charge densities remain constant at all distances $z$ outside the pulse. Therefore, the field $\Delta E_{z}(\mathbf{r}, t)$ under consideration is actually independent of $z$ outside the beam pulse, and schematically looks like as shown in Fig. 2.

According to (3), the longitudinal electric field is given as a summation of the longitudinal field term (18) and the superposition of all TM modes. Expanding the longitudinal current density $\tilde{J}_{z}(\mathbf{r}, f)$ in terms of the complete set of eigenmodes $\tilde{\mathcal{E}}_{q_{z}}\left(\mathbf{r}_{\perp}\right)$,

$$
\left.\tilde{J}_{z}(\mathbf{r}, f)=\sum_{q} \frac{w \epsilon k_{z_{q}}}{k_{\perp_{q}}^{2}} \mathcal{N}_{q}\left\langle\tilde{\mathcal{E}}_{q_{z}}\right| \tilde{J}_{z}\right) \tilde{\mathcal{E}}_{q_{z}}\left(\mathbf{r}_{\perp}\right),
$$

the expression for the longitudinal electric field can be presented as a modal expansion:

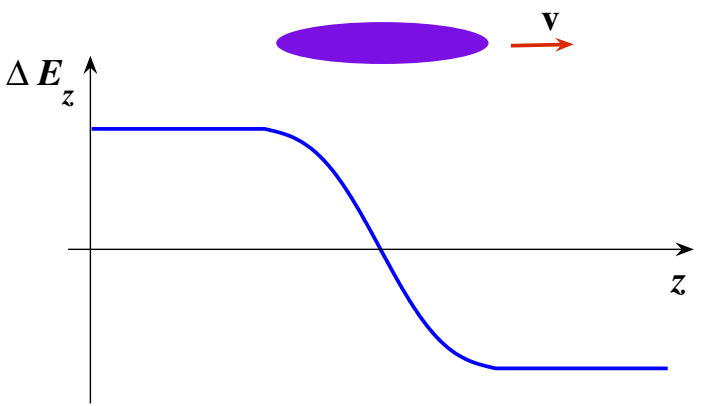

FIG. 2. (Color) Schematic illustration of the longitudinal field term (18).

$$
\begin{aligned}
\tilde{E}_{z}(\mathbf{r}, f)= & \sum_{q}\left[C_{+q}(z, f) e^{-j k_{z q} z}-C_{-q}(z, f) e^{+j k_{z q} z}\right. \\
& \left.-\frac{k_{z_{q}}}{j k_{\perp_{q}}^{2}} \mathcal{N}_{q}\left\langle\tilde{\mathcal{E}}_{q_{z}} \mid \tilde{J}_{z}\right\rangle\right] \tilde{\mathcal{E}}_{q z}\left(\mathbf{r}_{\perp}\right) .
\end{aligned}
$$

\section{THE ELECTRON BEAM DYNAMICS}

The state of a particle $i$ is described by a six-components vector, which consists of the particle's position coordinates $\mathbf{r}_{i}=\left(\mathbf{r}_{\perp i}, z_{i}\right)$ and velocity vector $\mathbf{v}_{i}$. The velocities of the particles, in the presence of electric $\mathbf{E}(\mathbf{r}, t)$ and magnetic $\mathbf{B}(\mathbf{r}, t)=\mu \mathbf{H}(\mathbf{r}, t)$ fields, are found from the Lorentz force equation:

$$
\frac{d \mathbf{v}_{i}}{d z}=-\frac{e}{m} \frac{\mathbf{E}\left(\mathbf{r}_{i}, t_{i}\right)+\mathbf{v}_{i} \times \mathbf{B}\left(\mathbf{r}_{i}, t_{i}\right)}{\gamma_{i} v_{z_{i}}}-\frac{\mathbf{v}_{i}}{\gamma_{i}} \frac{d \gamma_{i}}{d z},
$$

where $e$ and $m$ are the electron charge and mass, respectively, and the Lorentz relativistic factor $\gamma_{i}$ of each particle is found from the equation for kinetic energy:

$$
\frac{d \gamma_{i}}{d z}=-\frac{e}{m c^{2}} \frac{1}{v_{z_{i}}} \mathbf{v}_{i} \cdot \mathbf{E}\left(\mathbf{r}_{i}, t_{i}\right)
$$

The fields in Eqs. (23) and (24), represent the total (DC and AC) forces operating on a particle, and include also the self-field due to space charge.

The current distribution is determined by the positions $\mathbf{r}_{\perp i}$, the times $t_{i}(z)$, and the velocities $\mathbf{v}_{i}$ of the particles in the beam:

$$
\mathbf{J}(\mathbf{r}, t)=-\frac{Q}{N} \sum_{i=1}^{N} \frac{\mathbf{v}_{i}}{v_{z_{i}}} \delta\left(\mathbf{r}_{\perp}-\mathbf{r}_{\perp i}\right) \delta\left[t-t_{i}(z)\right] .
$$

Here $Q$ is the total charge of the e-beam pulse, and

$$
t_{i}(z)=t_{0_{i}}+\int_{0}^{z} \frac{d z^{\prime}}{v_{z_{i}}\left(z^{\prime}\right)}
$$

is the time it takes the $i$ th particle to arrive at a position $z$ $\left(t_{0 i}\right.$ is the time when the particle entered at $z=0$ ). In the positive-frequency domain, the current density of the beam is given by 


$$
\begin{aligned}
\tilde{\mathbf{J}}(\mathbf{r}, f) & =2 \int_{-\infty}^{+\infty} \mathbf{J}(\mathbf{r}, t) e^{+j w t} d t \\
& =-2 \frac{Q}{N} \sum_{i=1}^{N} \frac{\mathbf{v}_{i}}{v_{z_{i}}} \delta\left(\mathbf{r}_{\perp}-\mathbf{r}_{\perp i}\right) e^{+j w t_{i}(z)}
\end{aligned}
$$

and the excitation equation (5) can be rewritten as follows:

$$
\begin{aligned}
\frac{d}{d z} C_{ \pm q}(z, f)= & \pm \frac{1}{\mathcal{N}_{q}} \frac{Q}{N} \sum_{i=1}^{N} \\
& \times\left\{\frac{Z_{q}}{Z_{q}^{*}} \frac{\mathbf{v}_{\perp_{i}} \cdot \tilde{\mathbf{E}}_{ \pm q_{\perp}}^{*}\left(\mathbf{r}_{\perp i}\right)}{v_{z_{i}}}+\tilde{\mathcal{E}}_{ \pm q_{z}}^{*}\left(\mathbf{r}_{\perp i}\right)\right\} \\
& \times \exp \left[j\left(w t_{i}(z) \mp k_{z_{q}} z\right)\right] .
\end{aligned}
$$

The resulted expression together with the field presentation (1), (3), and (4), and with the beam dynamics equations (23), (24), and (26), form a close set of equations, enabling a self-consistent solution for the electromagnetic fields (radiation and space charge) and the beam trajectory in free-electron devices. Note that, after substitution of the current density (25) in (18), the longitudinal field term can be given in the form

$$
\Delta E_{z}(\mathbf{r}, t)=\frac{1}{2 \epsilon} \frac{Q}{N} \sum_{i=1}^{N} \delta\left(x-x_{i}\right) \delta\left(y-y_{i}\right) \operatorname{sgn}\left[t-t_{i}(z)\right]
$$

where $\operatorname{sgn}(t)=|t| / t$ is the sign function.

\section{SINGLE CHARGE}

To demonstrate the applicability of the modal expansion (22) for evaluation of longitudinal electric space-charge fields, we consider here a single pointlike charge, moving along a waveguide of length $L$ with a constant velocity $v_{z}$. Supposing no electromagnetic waves are entering the waveguide through its open ends, i.e. $C_{+q}(z=0, f)=0$ and $C_{-q}(z=L, f)=0$, the field amplitudes $C_{ \pm q}(z, f)$ can be found analytically:

$$
\begin{aligned}
& C_{+q}(z, f)=Q \tilde{\mathcal{E}}_{q z}^{*}\left(\mathbf{r}_{\perp_{p}}\right)\left(1-e^{-j \theta_{+q} z}\right) /\left(j \theta_{+q} \mathcal{N}_{q}\right) \\
& C_{-q}(z, f)=Q \tilde{\mathcal{E}}_{q z}^{*}\left(\mathbf{r}_{\perp_{p}}\right)\left(e^{-j \theta_{-q} L}-e^{-j \theta_{-q} z}\right) /\left(j \theta_{-q} \mathcal{N}_{q}\right) .
\end{aligned}
$$

Here $\mathbf{r}_{\perp p}$ is transverse position of the particle, and $\theta_{ \pm q}=$ $w / v_{z} \mp k_{z q}$.

As a numerical example, we consider here a point charge of $1 \mathrm{nC}$, moving with kinetic energy of $5.5 \mathrm{MeV}$ along a rectangular $a \times b=15 \times 10 \mathrm{~mm}^{2}$ waveguide of length $L=10 \mathrm{~cm}$. The parameters are typical to free-electron lasers operating in the millimeter wavelengths and the $\mathrm{THz}$ regime [12]. The interaction length $L$ was shortened to several centimeters for simplification of the numerical calculations. Figure 3 demonstrates the frequency depen-
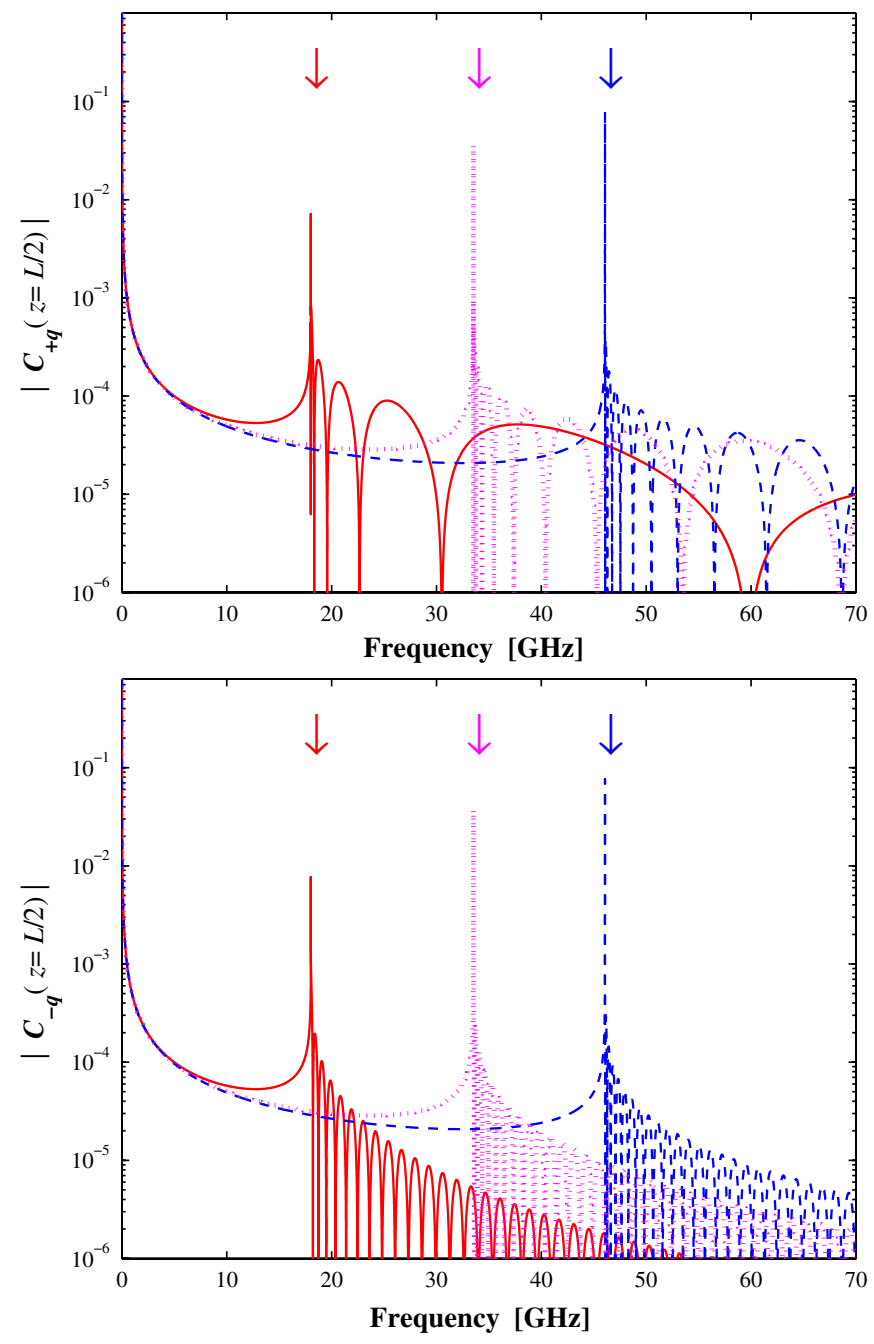

FIG. 3. (Color) Frequency dependence of the forward (top) and the backward (bottom) field amplitudes (30) found at the point $z=L / 2$ (only the first three modes are shown). Arrows show the cutoff frequencies of the modes.

dence of the field amplitudes (30) obtained in this case. The field is considered at the moment when the charge crosses the plain $z=L / 2$. Both forward and backward waves are found to play a comparative role in the calculations, dominating in the vicinity of the cutoff of each mode, and at zero frequency. Longitudinal dependence of the field amplitude $C_{ \pm q}(z, f)$ of $\mathrm{TM}_{11}$ is shown in Fig. 4 as a function of the relative longitudinal position $\Delta z=z-z_{p}$ in the vicinity of the cutoff frequency of the mode $f_{c o} \approx 18 \mathrm{GHz}$.

Substitution of the field amplitudes (30) into (3) and (4) results in the following tree-terms analytical expression for the longitudinal electric field:

$$
E_{z}(\mathbf{r}, t)=\sum_{\mathrm{TM}_{+q}}\left\{E_{z q}^{(p r)}(\mathbf{r}, t)+E_{z q}^{(c o)}(\mathbf{r}, t)+\Delta E_{z q}(\mathbf{r}, t)\right\}
$$

where 


$$
\begin{aligned}
E_{z q}^{(p r)}(\mathbf{r}, t)= & \frac{Q k_{\perp q}^{2}}{2 \pi \epsilon} \frac{\tilde{\mathcal{E}}_{q z}^{*}\left(\mathbf{r}_{\perp p}\right) \tilde{\mathcal{E}}_{q z}\left(\mathbf{r}_{\perp}\right)}{\left\langle\tilde{\mathcal{E}}_{q z} \mid \tilde{\mathcal{E}}_{q z}\right\rangle}\left\{\int_{c k_{\perp q}}^{\infty} \frac{\sin \left(w t-k_{z q} z\right)}{\left(w / v_{z}\right)^{2}-k_{z q}^{2}}\left(\frac{1}{k_{z q} v_{z}}-\frac{1}{w}\right) d w\right. \\
& \left.-\int_{c k_{\perp q}}^{\infty} \frac{\sin \left(w\left[t-L / v_{z}\right]-k_{z q}[L-z]\right)}{\left(w / v_{z}\right)^{2}-k_{z q}^{2}}\left(\frac{1}{k_{z q} v_{z}}-\frac{1}{w}\right) d w-2 \int_{c k_{\perp q}}^{\infty} \frac{\sin \left(w\left[t-z / v_{z}\right]\right)}{\left(w / v_{z}\right)^{2}-k_{z q}^{2}} \frac{d w}{w}\right\}
\end{aligned}
$$

is the field component corresponding to propagating waves, and

$$
\begin{aligned}
E_{z q}^{(c o)}(\mathbf{r}, t)= & \frac{Q k_{\perp q}^{2}}{2 \pi \epsilon} \frac{\tilde{\mathcal{E}}_{q z}^{*}\left(\mathbf{r}_{\perp p}\right) \tilde{\mathcal{E}}_{q z}\left(\mathbf{r}_{\perp}\right)}{\left\langle\tilde{\mathcal{E}}_{q z} \mid \tilde{\mathcal{E}}_{q z}\right\rangle}\left\{\int_{0}^{c k_{\perp q}} \frac{e^{-\left|k_{z q}\right| z}}{\left(w / v_{z}\right)^{2}+\left|k_{z q}\right|^{2}}\left[\frac{\cos (w t)}{\left|k_{z q}\right| v_{z}}-\frac{\sin (w t)}{w}\right] d w\right. \\
& \left.-\int_{0}^{c k_{\perp q}} \frac{e^{-\left|k_{z q}\right|(L-z)}}{\left(w / v_{z}\right)^{2}+\left|k_{z q}\right|^{2}}\left[\frac{\cos \left(w\left[t-L / v_{z}\right]\right)}{\left|k_{z q}\right| v_{z}}-\frac{\sin \left(w\left[t-L / v_{z}\right]\right)}{w}\right] d w-2 \int_{0}^{c k_{\perp q}} \frac{\sin \left(w\left[t-z / v_{z}\right]\right)}{\left(w / v_{z}\right)^{2}+\left|k_{z q}\right|^{2}} \frac{d w}{w}\right\}
\end{aligned}
$$

corresponds to cutoff modes. The third term is the longitudinal field, given by

$$
\Delta E_{z q}(\mathbf{r}, t)=\frac{Q}{2 \epsilon} \frac{\tilde{\mathcal{E}}_{q z}^{*}\left(\mathbf{r}_{\perp p}\right) \tilde{\mathcal{E}}_{q z}\left(\mathbf{r}_{\perp}\right)}{\left\langle\tilde{\mathcal{E}}_{q z} \mid \tilde{\mathcal{E}}_{q z}\right\rangle} \operatorname{sgn}\left(t-z / v_{z}\right) .
$$
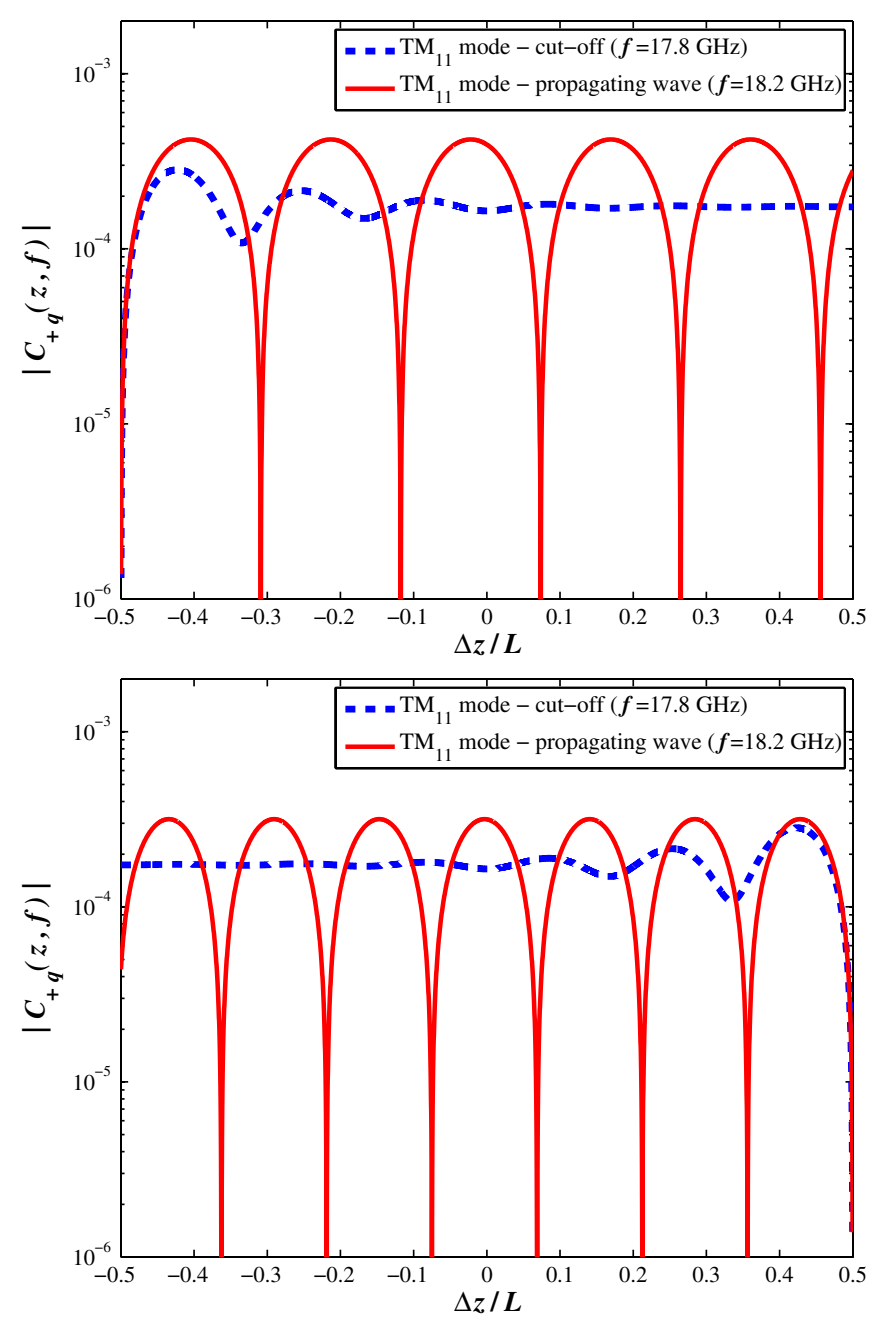

FIG. 4. (Color) Spatial (longitudinal) dependence of the forward (top) and backward (bottom) field amplitude (30) of $\mathrm{TM}_{11}$ mode in the vicinity of the cutoff.
Figure 5 demonstrates the field (31) found at the waveguide axis at the moment when the charge passes the plain $z=L / 2$. The results are shown as obtained in the calculations with a single $\mathrm{TM}_{11}$ mode and with $5050 \mathrm{TM}_{m, n}$ modes $(m+n \leq 200)$ taken into account. Inspection of Fig. 5 reveals that, at long distances from the charge, the steplike longitudinal field term (18) is mainly compensated
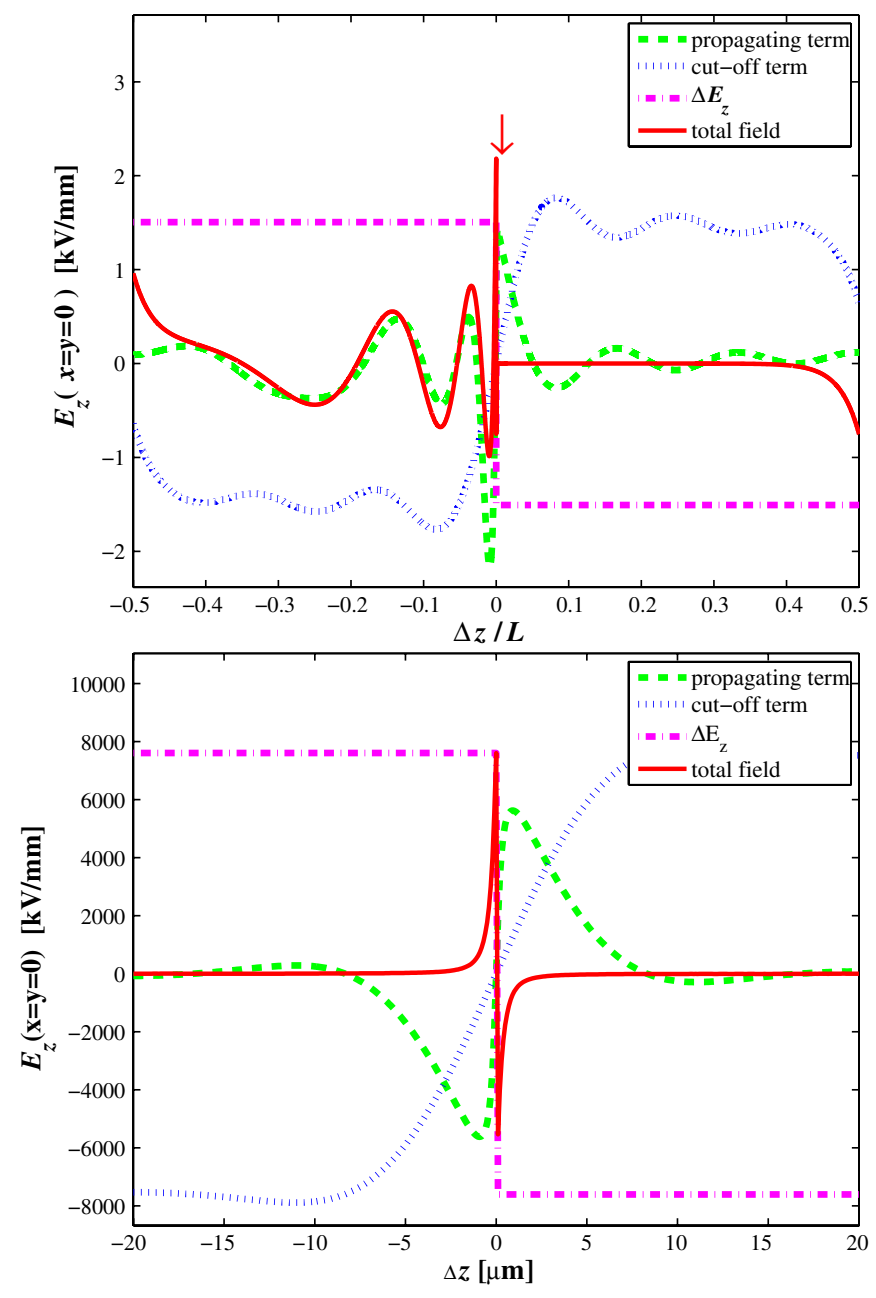

FIG. 5. (Color) Longitudinal electric field (31) obtained with a single $\mathrm{TM}_{11}$ mode (top) and with $5050 \mathrm{TM}_{m, n}$ modes (bottom). 


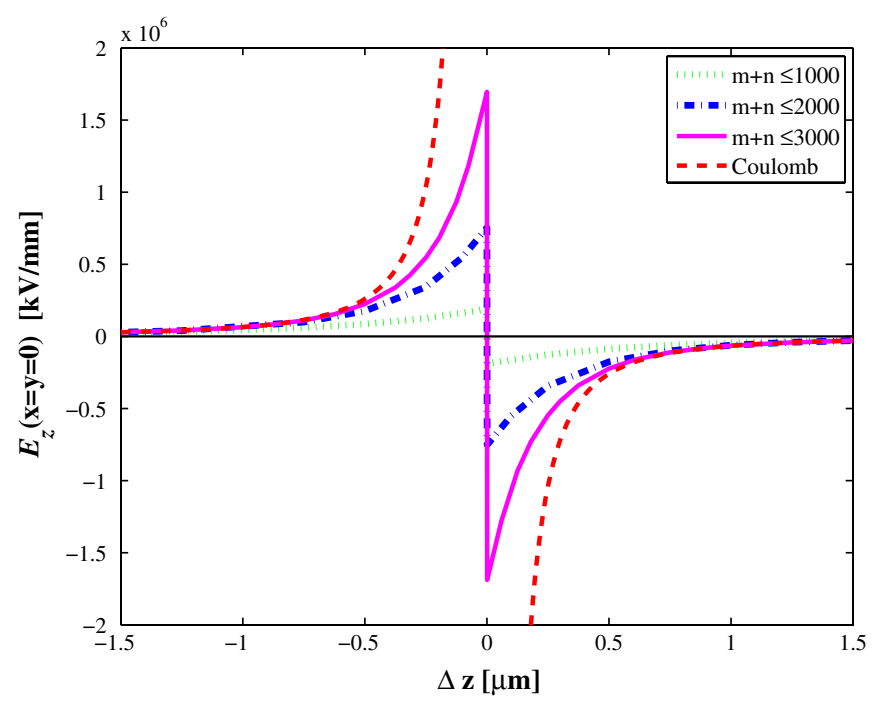

FIG. 6. (Color) Longitudinal electric field (31).

by the field component originated from the cutoff modes, while the propagating waves seem to play an important role at short distances from the charge. Convergence of the calculations is found to be extremely slow as demonstrated in Fig. 6. We note that the total electric field found far of the waveguide's ends after the summation of all the modes is of a short range, and it seems to be insensitive to the waveguide's length $L$; then the dependence of the total field obtained inside the waveguide on the length $L$ is considered as an artificial.

\section{STATIC POINTLIKE CHARGE IN A WAVEGUIDE}

The Coulomb field given in Fig. 6 for a comparison was obtained by Lorenz transformation of the free-space Coulomb field of a point static charge

$$
\mathbf{E}^{(C)}(\mathbf{r})=\frac{Q}{4 \pi \epsilon} \frac{\mathbf{r}-\mathbf{r}_{p}}{\left|\mathbf{r}-\mathbf{r}_{p}\right|^{3}}
$$

from its rest frame to the laboratory system. Note that this field does not fulfill the boundary conditions at the waveguide walls. Nevertheless, the resulted field is supposed to be a good approximation in the vicinity of the waveguide axis.

However, boundary conditions should be introduced in calculations of the field far of the waveguide axis. To find an analytic expression for the field, we first consider a static pointlike charge in a point $\mathbf{r}_{p}$ inside a waveguide. The electric field of the charge can be found as a solution of Poisson equation

$$
\nabla \cdot \mathbf{E}(\mathbf{r})=\frac{Q}{\epsilon} \delta\left(\mathbf{r}-\mathbf{r}_{p}\right)
$$

imposing the proper boundary conditions. This static solution can be expressed as an expansion over the wave- guide eigenmodes, taken at zero frequency:

$$
\mathbf{E}(\mathbf{r})=\left.\sum_{ \pm q} \alpha_{ \pm q}(z) \tilde{\mathbf{E}}_{ \pm q}\left(\mathbf{r}_{\perp}\right)\right|_{f=0} .
$$

Substitution of the expansion (37) into Eq. (36) and use of the orthogonality of the transverse eigenmodes $\tilde{\mathbf{E}}_{ \pm q}\left(\mathbf{r}_{\perp}\right)$ result in the following differential equation for the expansion coefficients $\alpha_{ \pm q}(z)$ :

$$
\begin{aligned}
& \frac{d \alpha_{+q}}{d z}+k_{\perp q} \alpha_{+q}(z)-\frac{d \alpha_{-q}}{d z}+k_{\perp q} \alpha_{-q}(z) \\
& =\frac{Q}{\epsilon} \frac{\tilde{\mathcal{E}}_{q_{z}}\left(\mathbf{r}_{\perp}\right)}{\left\langle\tilde{\mathcal{E}}_{q z} \mid \tilde{\mathcal{E}}_{q z}\right\rangle} \delta\left(z-z_{p}\right) .
\end{aligned}
$$

Solution of this differential equation, while fulfilling the obvious symmetry condition $E_{z}\left(\mathbf{r}_{\perp},\left[z_{p}+\Delta z\right]\right)=$ $-E_{z}\left(\mathbf{r}_{\perp},\left[z_{p}-\Delta z\right]\right)$, can be presented in the form

$$
\begin{aligned}
& \alpha_{+q}(z)=\frac{Q}{2 \epsilon} \frac{\tilde{\mathcal{E}}_{q_{z}}\left(\mathbf{r}_{p \perp}\right)}{\left\langle\tilde{\mathcal{E}}_{q z} \mid \tilde{\mathcal{E}}_{q z}\right\rangle} e^{-k_{\perp q}\left(z-z_{p}\right)} u\left(z-z_{p}\right) \\
& \alpha_{-q}(z)=\frac{Q}{2 \epsilon} \frac{\tilde{\mathcal{E}}_{q_{z}}\left(\mathbf{r}_{p \perp}\right)}{\left\langle\tilde{\mathcal{E}}_{q z} \mid \tilde{\mathcal{E}}_{q z}\right\rangle} e^{+k_{\perp q}\left(z-z_{p}\right)} u\left(z_{p}-z\right) .
\end{aligned}
$$

Here $u(z)$ is the step function. Note that according to (39), only TM modes should be included in the summation in Eq. (37).

Substituting the coefficients (39) into the expansion (37), the electric field of a static charge in a waveguide can finally be given as follows:

$$
\begin{aligned}
\mathbf{E}(\mathbf{r})= & \frac{Q}{2 \boldsymbol{\epsilon}} \sum_{\mathrm{TM}_{q}} \frac{\tilde{\mathcal{E}}_{q_{z}}\left(\mathbf{r}_{p \perp}\right)}{\left\langle\tilde{\mathcal{E}}_{q z} \mid \tilde{\mathcal{E}}_{q z}\right\rangle} e^{-k_{\perp q}\left|z-z_{p}\right|}\left\{\left.u\left(z-z_{p}\right) \tilde{\mathbf{E}}_{+q}\left(\mathbf{r}_{\perp}\right)\right|_{f=0}\right. \\
& \left.+\left.u\left(z_{p}-z\right) \tilde{\mathbf{E}}_{-q}\left(\mathbf{r}_{\perp}\right)\right|_{f=0}\right\} .
\end{aligned}
$$

This expression enables more effective field evaluation in comparison with direct calculations according to (31)(34); this is because of the simple analytic form which does not include, in contrast to the direct calculations, integrals of oscillating functions arising due to the inverse Fourier transform. More of this, exponential term $e^{-k_{\perp q}\left|z-z_{p}\right|}$ in Eq. (40) provides a much faster convergence of the calculations with a number of the eigenmodes taken into account, depending on the relative distance $\left|z-z_{p}\right|$ considered.

Figure 7 presents the field obtained after Lorenz transformation of (40) to the laboratory frame. The field is found to be very close to the Coulomb one at the vicinity of the waveguide axis and at small $\left|z-z_{p}\right|$ [see Fig. 7(a)]. However, it falls much faster than the Coulomb field at long distances as shown in Fig. 7(b). Figure 7(c) demonstrates the effects of the waveguide walls on the field. 

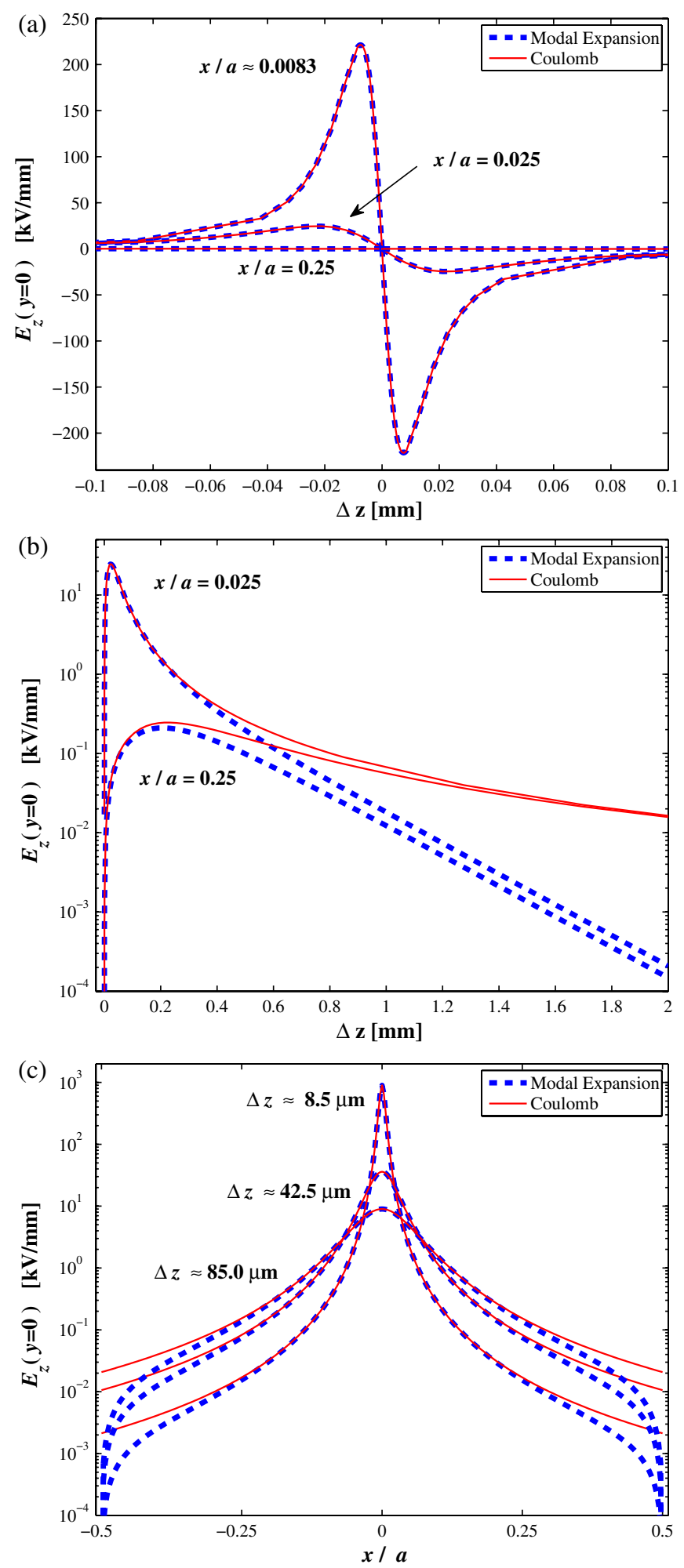

FIG. 7. (Color) Longitudinal electric field of a $1 \mathrm{nC}$ charge, moving with a constant $5.5 \mathrm{MeV}$ kinetic energy along a rectangular waveguide.

\section{CONCLUSIONS}

The space-frequency model is found to satisfy the full set of Maxwell's equations, including Gauss's laws for electric and magnetic fields. Expressions describing the longitudinal electric field in the model include, in addition to a simple steplike one-dimensional term, summation over both forward and backward waves, which contributes mainly at zero frequency and near cutoff. None of these terms can be neglected in the calculations of the spacecharge field. The model enables a direct evaluation of longitudinal space-charge fields associated with an electron beam in a waveguide according to Eq. (3) or Eq. (22). Nevertheless, such direct calculations demonstrate an extremely slow convergence even in a simple case of a single pointlike charge.

To improve the efficiency of the method, analytical 3D approximation based on Lorenz-transformed fields (40) is suggested. Application of Lorenz-transformed Coulomb free-space field is found to be sufficient and reasonable in configurations where the electron beam does not approach the waveguide's walls.

[1] Y. Pinhasi and Yu. Lurie, Phys. Rev. E 65, 026501 (2002).

[2] E. L. Saldin, E. A. Schneidmiller, and M. V. Yurkov, Nucl. Instrum. Methods Phys. Res., Sect. A 528, 355 (2004).

[3] T. Shaftan and Z. Huang, Phys. Rev. ST Accel. Beams 7, 080702 (2004).

[4] J. H. Wu, Z. R. Huang, and P. Emma, Phys. Rev. ST Accel. Beams 11, 040701 (2008).

[5] N. Marcuvitz and J. Schwinger, J. Appl. Phys. 22, 806 (1951).

[6] Y. Pinhasi, Yu. Lurie, and A. Yahalom, Phys. Rev. E 71, 036503 (2005).

[7] Y. Pinhasi, Y. Lurie, and A. Yahalom, Nucl. Instrum. Methods Phys. Res., Sect. A 528, 62 (2004).

[8] Yu. Lurie and Y. Pinhasi, Phys. Rev. ST Accel. Beams 10, 080703 (2007).

[9] Y. Pinhasi and A. Gover, Phys. Rev. E 48, 3925 (1993).

[10] A. Gover and E. Dyunin, Phys. Rev. Lett. 102, 154801 (2009).

[11] E. Hemsing et al., Phys. Rev. Lett. 102, 174801 (2009).

[12] E. Dyunin, Yu. Lurie, Y. Pinhasi, A. Gover, and H. Marks, in Proceedings of IEEE 25th Convention of Electrical \& Electronics Engineers in Israel (IEEEI 2008), http:// dx.doi.org/10.1109/EEEI.2008.4736655, p. 825. 